\section{Efeitos de diferentes intensidades de treinamento aeróbio sobre a lipemia de adolescentes obesos}

\section{Effects of different intensities of aerobic training on lipemia in obese adolescents}

Camila Tenório Calazans de Lira

Crivaldo Gomes Cardoso Júnior ${ }^{2}$

Priscyla Praxedes Gomes ${ }^{1}$

Thiago Ricardo dos Santos Tenório ${ }^{1}$

Mara Cristina Lofrano do Prado ${ }^{3}$

Moacir de Novaes Lima Ferreira ${ }^{4}$

Wagner Luiz do Prado ${ }^{1}$

\section{Resumo}

O objetivo do presente estudo foi verificar o efeito de diferentes intensidades de treinamento físico aeróbio sobre a lipemia de adolescentes obesos submetidos à intervenção multidisciplinar. Quarenta e três adolescentes (idade $=15,25 \pm 1,26$ ) obesos (índice de massa corporal [IMC] acima do percentil $95\left(\right.$ CDC) $\left(\mathrm{IMC}=34,48 \pm 3,88 \mathrm{~kg} / \mathrm{m}^{2}\right)$ de ambos os gêneros foram alocados aleatoriamente em dois grupos: treinamento de alta intensidade (TAI) - limiar ventilatório I (LVI); e treinamento de baixa intensidade (TBI) - 20\% abaixo do LVI. As sessões de exercício $(3 \mathrm{x} / \mathrm{semana})$ foram isocalóricas $(350 \mathrm{kcal})$. Todos os adolescentes receberam a mesma intervenção nutricional, psicológica e clínica durante 24 semanas. Foram determinados os níveis de triglicerídeos, colesterol total e frações, antes e após a intervenção. Para comparações entre os grupos e momentos utilizou-se ANOVA two-way com Post-hoc de Fisher. Após 24 semanas observou-se redução no colesterol total $(9 \%$ - p<0,05) e LDL $(17,7 \%$ - p<0,05) e elevação do HDL $(22,8 \%$ - $\mathrm{p}<0,05)$ em resposta ao TBI. O TAI foi efetivo na redução do LDL $(14,3 \%$ - $\mathrm{p}<0,05)$ e aumento no HDL $(28,1 \%$ - $\mathrm{p}<0,05)$. As razões lipídicas Colesterol Total/HDLcolesterol, LDL-colesterol/HDL-colesterol (Índices de Castelli I e II), reduziram em ambos os grupos: TBI (-24,81\% Castelli I), (-33,59\% Castelli II); TAI (- 24,45\% Castelli I), (-31,83\% Castelli II). Os resultados sugerem que ambas intensidades de treinamento físico são capazes de promover alterações positivas na lipemia de adolescentes obesos submetidos a intervenção multidisciplinar, entretanto, quando comparado ao treinamento de alta intensidade, o treinamento de baixa intensidade é mais efetivo na redução do colesterol total.

\section{Palavras-chave}

Obesidade, Dislipidemia, Exercício.

\begin{abstract}
The aim of this study was to investigate the effect of different intensities of aerobic exercise on lipemia in obese adolescents submitted to a multidisciplinary intervention. Forty-three obese (body mass index [BMI] higher than 95th percentile $(C D C)(B M I=34.48 \pm 3.88 \mathrm{~kg} / \mathrm{m} 2)$ adolescents (age $=15.25 \pm$ 1.26) from both genders were randomly allocated into two groups: high intensity training (HIT) - ventilatory threshold (LVI), and low intensity training (LIT) - $20 \%$ below the LVI. Exercise sessions ( $3 x$ week) were isocaloric (350 kcal). All adolescents received the same nutritional, psychological and clinical counseling for 24 weeks. Were determined levels of triglycerides, total cholesterol and fractions, before and after the intervention. Comparisons between groups and times were made by two-way ANOVA with Fisher as Post-hoc. After 24 weeks there was a reduction in total cholesterol $(9 \%-p<0.05)$ and LDL $(17.7 \%-p<0.05)$ and increasing in HDL $(22.8 \%-. p<0.05)$ in response to LIT. HIT was effective to decrease $L D L(14.3 \%-p<0.05)$ and increase HDL $(28.1 \%-. p<0.05)$. The lipid ratios: total cholesterol / HDL - cholesterol; LDL-colesterol/HDL-colesterol (Castelli Indices I and II), decreased in both groups: LIT (-24.81\% Castelli I), (II -33.59\% Castelli); (HIT-24.45\% Castelli I), (-31,83 $\%$ Castelli II). The results suggest that both intensities of exercise training can promote positive changes in lipid profile in obese adolescents submitted to a multidisciplinary intervention, however when compared to bigh-intensity training, low intensity training is more effective in reducing total cholesterol.
\end{abstract}

\section{Keywords}

Obesity, Dyslipidemia, Exercise.
Rev Bras Ativ Fis Saúde p. 761-770 DOI:

http://dx.doi.org/10.12820/rbafs.v.18n6p761

${ }^{1}$ Universidade de Pernambuco, Programa Associado de Pós-graduação em Educação Física UPE/UFPB, Recife, Pernambuco, Brasil.

2Universidade Estadual de Londrina, Centro de Educação Física e Esporte, Londrina, Paraná, Brasil.

3Universidade de Pernambuco, Bolsista PNPD Faculdade de Odontologia, Recife, Pernambuco, Brasil.

4Universidade de Pernambuco, Unidade de Pesquisa Clínica Hospital Universitário Oswaldo Cruz, Recife, Pernambuco, Brasil.

Obesity, Dyslipidemia, Exercise. 


\section{INTRODUÇÃO}

A obesidade é uma doença complexa e de etiologia multifacetada que pode ter seu início já nos primeiros anos de vida ${ }^{1}$. No Brasil, considerando apenas a população de adolescentes, estima-se que a obesidade esteja presente em aproximadamente $14 \%$ deles $^{2}$, o que faz com que a estimativa de obesidade na população de adolescentes brasileiros seja ainda mais preocupante.

Embora a obesidade seja um potente preditor de risco para o desenvolvimento de doenças cardiovasculares em fases mais tardias da vida humana ${ }^{3}$, parte de seus desfechos clínicos negativos ocorrem já na adolescência, como é o caso da precipitação dos distúrbios nos lípides sanguíneos. Sabe-se que prevalência de dislipidemia em crianças e adolescentes varia no mundo todo entre 2,9 e 33\%, adotando o ponto de corte de $200 \mathrm{mg} / \mathrm{dL}$ para os níveis de colesterol total (CT $)^{4}$. No Brasil, a prevalência situa-se entre 28 e $40 \%$ das crianças e adolescentes, quando o critério adotado é o CT superior a $170 \mathrm{mg} / \mathrm{dL}^{5}$.

Esta alta prevalência de dislipidemia fica ainda mais preocupante quando a obesidade está agregada, o que justifica por associação positiva entre indicadores de adiposidade com o diagnóstico de dislipidemia ${ }^{1}$. Diante disto, são prementes ações de combate à dislipidemia, sobretudo em adolescentes obesos que, naturalmente, são mais propensos aos desfechos clínicos negativos desta associação.

A prática insuficiente de exercício físico se destaca dentre os principais fatores que imprimem a crescente ascendência à dislipidemia entre os adolescentes ${ }^{6}$. Em que pese a carência de investigações na população de adolescentes, sabe-se que adultos fisicamente ativos apresentam maior concentração plasmática de HDLcolesterol, menores concentrações de LDL-colesterol e triglicérides quando comparados aos seus pares sedentários ${ }^{7}$. Entretanto, tem sido verificado que a ausência de prática de exercícios físicos na infância e adolescência, mais do que na vida adulta, precipita o surgimento de dislipidemia na idade adulta ${ }^{8}$, em contrapartida estudos têm verificado melhorias no perfil lipídico com treinamento físico aeróbio ${ }^{9,10}$.

Com base nisto, a IV Diretriz Brasileira de Prevenção a Aterosclerose ${ }^{11}$ recomenda que a mudança de estilo de vida deva ser a primeira meta terapêutica de combate à dislipidemia. Assim, a prática regular e sistematizada do exercício físico deve ser encorajada na prevenção primária na história natural da doença aterosclerótica, dentre outros aspectos, por conta de seus efeitos benéficos de combate à dislipidemia.

Ademais, sabe-se que a intensidade empregada na prática pode exercer um papel determinante no desfecho induzido pelo exercício. $\mathrm{Na}$ revisão sistemática de Tambalis et al. ${ }^{12}$ foi verificado que apenas os programas de exercício aeróbico de alta intensidade resultaram em efeitos mais favoráveis nas lipoproteínas circulantes. Entretanto, não foi considerado o efeito do maior dispêndio energético induzido pelo exercício de maior intensidade, de modo que o efeito real da intensidade do treinamento físico, sobretudo em adolescentes, ainda não foi determinado.

Desta maneira o presente estudo objetivou verificar o efeito de diferentes intensidades de treinamento físico aeróbio sobre o perfil lipídico e lipoprotéico de adolescentes obesos submetidos a intervenção multidisciplinar.

\section{MÉTODOS}

\section{Amostra}

Adolescentes obesos foram recrutados através de divulgação na mídia local. Os critérios de inclusão adotados foram idade entre 13 e 18 anos, púberes (estágios 
3 e 4 de Tanner) ${ }^{13}$, massa corporal inferior a $120 \mathrm{~kg}$, índice de massa corporal (IMC) acima do percentil 95 (CDC) $(2000)^{14}$, ausência de hipertensão e/ou outras doenças metabólicas, ambos avaliados por um médico endocrinologista e que referendou à prontidão à prática regular de exercícios físicos. Todos os participantes foram triados quanto à sua motivação intrínseca à prática e somente foram selecionados os adolescentes obesos que estavam positivamente motivados a esta modificação comportamental de intervenção multidisciplinar para tratamento da obesidade por 24 semanas. Foram excluídos os adolescentes obesos que faziam uso crônico de álcool ou tabagismo; uso contínuo de antiinflamatório ou outras drogas que alterassem o metabolismo; gravidez durante a intervenção; e, frequência inferior a $75 \%$ a qualquer uma das intervenções propostas.

Após serem esclarecidos sobre todos os procedimentos experimentais, os pais ou responsáveis legais assinaram termo de Consentimento Livre e Esclarecido. Este estudo foi aprovado pelo Comitê de Ética da Universidade de Pernambuco (n॰154/09).

\section{Desenho do estudo}

Inicialmente 229 adolescentes se voluntariam para participar do estudo. Durante a primeira visita foram realizadas as medidas antropométricas (massa corporal e estatura), determinado o estágio maturacional por meio de auto-avaliação, seguindo os critérios estabelecidos por Tanner ${ }^{13}$. Os adolescentes foram submetidos a uma entrevista semi-estruturada individual e confidencial contendo oito questões destinadas a avaliar motivos pessoais para a busca de tratamento de perda de peso e as barreiras para a perda de peso tendo sido realizada por um psicólogo em uma sala silenciosa. Cento e oitenta e seis voluntários não atenderam aos critérios de inclusão. $\mathrm{Na}$ segunda visita ao laboratório, os adolescentes foram submetidos à consulta clínica e realizaram eletrocardiograma de repouso para posterior triagem médica. Ao final, 43 adolescentes obesos (13 meninos e 30 meninas) foram incluídos na amostra. Após as avaliações preliminares, os participantes foram aleatoriamente alocados (utilizando-se o método coinfliping) no grupo treinamento de alta intensidade (TAI) ( $\mathrm{n}=20)$ ou grupo treinamento de baixa intensidade (TBI) $(\mathrm{N}=23)$. Todos os adolescentes receberam a mesma intervenção nutricional, psicológica e clínica (figura 1).

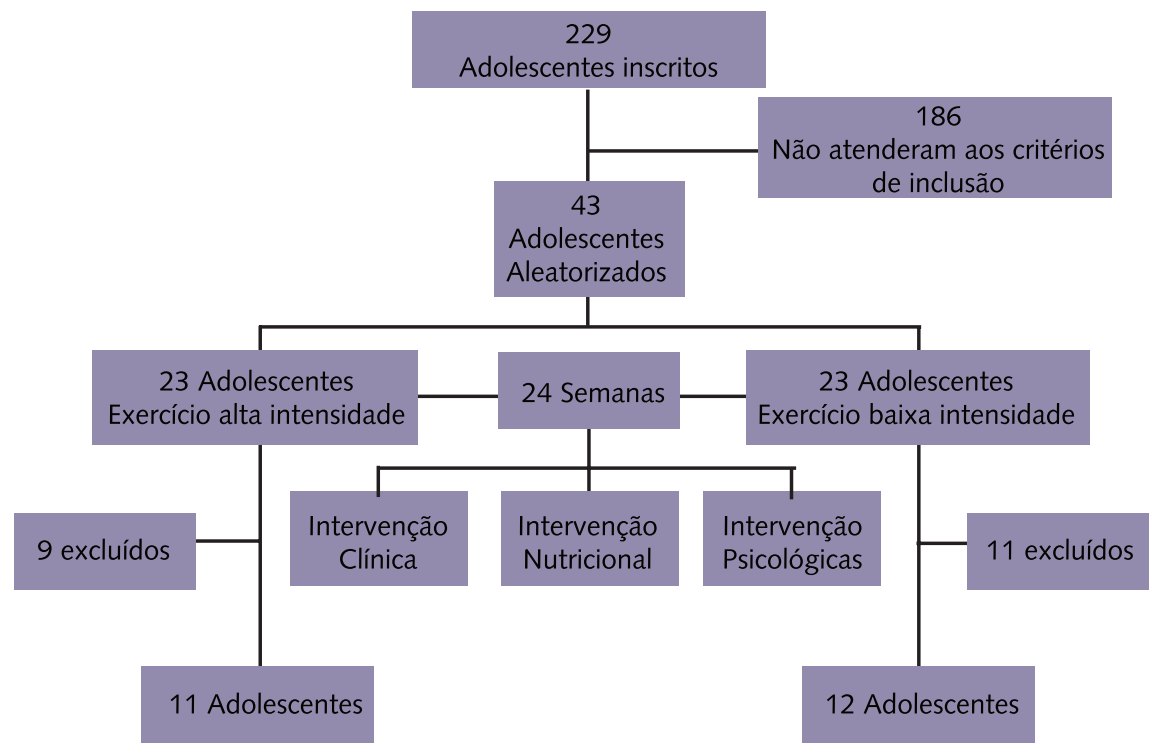

Figura 1 - Desenho do Estudo. 


\section{Intervenção clínica}

Os adolescentes recebiam acompanhamento clínico mensal, durante as consultas com o médico endocrinologista, o qual transmitia orientações sobre mudanças no estilo de vida e acompanhava a evolução clínica de cada voluntário. Entretanto, não houve prescrição de nenhum tipo de medicamento durante todo o protocolo experimental.

\section{Intervenção nutricional}

A intervenção nutricional foi realizada por meio de reuniões em grupos com aproximadamente nove adolescentes cada. Estes encontros tiveram periodicidade semanal, com duração de uma hora e nele foram abordados os seguintes temas: pirâmide alimentar, fast food, rotulagem nutricional, dietas da moda, nutrição na promoção de saúde, tipos de gordura (trans, saturada e insaturada), produtos diet e light e comportamento em ocasiões especiais (festas, aniversários, eventos sociais). Entretanto, é importante mencionar que não foi prescrita nenhuma dieta individualizada.

\section{Intervenção psicológica}

Foram realizados atendimentos psicológicos em grupos (aproximadamente nove adolescentes), uma vez por semana, durante uma hora, conduzidos pela psicóloga do grupo. Nestas sessões, foram realizadas dinâmicas que abordaram temas como emoções (sentimentos), auto-estima, imagem corporal, preconceito, transtornos alimentares, dificuldades, questões familiares, bem como questões que pudessem surgir no grupo. Além disto, a psicóloga ficava à disposição dos voluntários para apoio e acolhimento, se necessário.

\section{Treinamento Aeróbio}

Os participantes de ambos os grupos (TAI e TBI) realizaram treinamento aeróbio individualizado em esteira rolante, 3 vezes por semana, sob a supervisão de profissionais de Educação Física.

Os voluntários do TAI treinaram em intensidade de velocidade correspondente ao limiar ventilatório I (LVI), e os do TBI exercitaram-se em intensidade de velocidade correspondente a $20 \%$ abaixo do LVI. Para ambos os grupos as sessões foram isocalóricas, com o gasto energético fixado em $350 \mathrm{Kcal} /$ sessão, estimado por calorimetria indireta. Uma vez que cada voluntário treinava em uma carga específica, a duração das sessões diferiu entre os sujeitos, para garantir que o gasto energético pré-fixado fosse atingido, a duração de cada sessão de treinamento foi determinada considerando-se que para cada litro de oxigênio consumido $\left(\mathrm{VO}_{2}\right)$, $4,96 \mathrm{Kcal}$ de energia era liberada, baseado no equivalente metabólico da tarefa (MET), conforme a seguir:

$$
\text { Duração da sessão de exercício }(\mathrm{min})=350 \mathrm{Kcal} /\left(\mathrm{VO}_{2}(\mathrm{~L} / \mathrm{min} \text {.) na carga*1MET) }\right.
$$

Onde: $\mathrm{VO}_{2}=$ consumo de oxigênio; $M E T$ = equivalente metabólico da tarefa.

\section{Avaliações}

Todos os voluntários foram submetidos ao mesmo protocolo de avaliações, antes (basal) e após 24 semanas de intervenção multidisciplinar. As medidas eram realizadas sempre no mesmo período do dia, com o objetivo de minimizar quaisquer variações circadianas nas variáveis analisadas. 


\section{Medidas antropométricas e composição corporal}

As medidas de massa corporal (MC), estatura e circunferência de cintura (CC) foram realizadas em uma balança Filizola ${ }^{\circledR}$ com precisão de $0,1 \mathrm{~kg}$, um estadiômetro de madeira com precisão de 0,1 cm e fita métrica inextensível com precisão de 0,1 $\mathrm{cm}$. Em seguida foi calculado o índice de massa corporal (IMC), dividindo-se a massa corporal $(\mathrm{Kg})$ pelo quadrado da estatura $\left(\mathrm{m}^{2}\right)$.

As dobras cutâneas triciptal (TR), subescapular (SE) e da panturrilha medial (PA) foram mensuradas com um adipômetro de marca Lange ${ }^{\circledR}$ resolução de $0,1 \mathrm{~cm}$, no hemicorpo direito. Após três medidas, a média era calculada. $\mathrm{O}$ percentual de gordura $(\% \mathrm{G})$ foi estimado através da equação de Slaughter e cols. ${ }^{15}$. Todas as avaliações antropométricas foram realizadas por um único avaliador.

\section{Estágio Maturacional}

Cada participante recebeu desenhos dos cinco estágios do tamanho da mama, o desenvolvimento genital e púbico ${ }^{13}$. Em uma sala isolada, os adolescentes foram convidados a olhar para os desenhos, ler as descrições, pensar sobre como eles eram em comparação com os desenhos e escolher o que mais se assemelhava com eles. Para os homens, as imagens da genitália e dos pêlos pubianos foram classificados em cinco estágios de desenvolvimento. $\mathrm{O}$ mesmo ocorrendo para as imagens das mamas e dos pêlos pubianos dentre as mulheres.

\section{Determinação do limiar ventilatório}

$\mathrm{O} \mathrm{VO}_{2}$ foi determinado por meio da análise direta de gases, utilizando-se um analisador metabólico de circuito aberto (Cortex Biophysik Metalyzer IIB, Germany), durante teste contínuo e incremental em esteira rolante (Inbrasport Super ATL, Brazil). Após aquecimento de 3 minutos $(3 \mathrm{~km} / \mathrm{h})$, a velocidade era acrescida em $1 \mathrm{~km} / \mathrm{h}$ a cada minuto até a exaustão voluntária, ou quando a escala de Borg e o quociente respiratório apresentassem valores superiores a 18 e 1,15, respectivamente. A inclinação foi mantida constante em $1 \%$ durante todo o teste. Antes de cada teste, os instrumentos eram calibrados para a mistura de gases seguindo-se todas as instruções do fabricante.

O LVI foi determinado por meio da inspeção visual por dois pesquisadores independentes, como o ponto de quebra de linearidade entre a produção de dióxido de carbono $\left(\mathrm{CO}_{2}\right)$ e o $\mathrm{VO}_{2}$ (V-slope); ponto no qual a curva do equivalente ventilatório de oxigênio $\left(\mathrm{VE} / \mathrm{VO}_{2}\right)$ e a fração expirada de $\mathrm{CO}_{2}\left(\mathrm{P}_{\mathrm{ET}} \mathrm{CO}_{2}\right)$ atingiram seus menores valores, imediatamente antes do aumento dos respectivos equivalentes ${ }^{16}$.

\section{Análises bioquímicas}

A coleta de sangue foi realizada através de punção periférica na veia cubital, entre às 8:00 e 9:00h da manhã, após jejum noturno de 12 horas. Em seguida as alíquotas foram estocadas em tubos com anticoagulante (EDTA-K3E). As análises do colesterol total e fração HDL-c, e níveis de triglicérides foram realizados por espectrofotometria (Cobas Integra 400 Plus), com kits Roche ${ }^{\circledR}$. A fração LDL-c foi calculada de forma indireta através da fórmula de Friedewald: [LDL-c $=\mathrm{CO}$ LESTEROL TOTAL - HDL-c - (TRIGLICÉRIDES/5)] ${ }^{17}$. Para a análise de depuração do colesterol total e do LDLcolesterol, ambas mediadas pelos níveis de HDL colesterol, empregou-se o cálculo dos índices de Castelli I e II, respectivamente ${ }^{18}$, em que: índice de Castelli I: quociente entre os níveis de colesterol total pela concentração de HDL-c (CT/HDL-c); e o índice de Castelli II: quociente entre os níveis de LDL-c pela concentração de HDL-c (LDL-c/HDL-c). 


\section{Análise estatística}

Os dados foram analisados através do programa SPSS versão 20 e Statistica versão 8.0. A normalidade dos dados foi determinada através do teste de Shapiro Wilk, e os dados são apresentados por média e desvio padrão. Para a análise comparativa entre os grupos (TAI e TBI) e os momentos (pré e pós-intervenção) foi utilizada a análise de variância de dois caminhos para medidas repetidas (ANOVA - two way), com Post-hoc de Fisher. O nível de significância foi fixo em 5\%.

\section{RESULTADOS}

A amostra foi composta por 43 adolescentes (13 meninos e 30 meninas; $15,4 \pm 1,5$ anos; IMC: $34,4 \pm 4,3 \mathrm{~kg} / \mathrm{m}^{2}$ ), entretanto, durante a intervenção vinte e três voluntários foram excluídos, dos quais, nove do TAI (um por problemas ortopédicos e oito por aderência inferior a 75\%) e onze do TBI (aderência inferior a 75\%), desta forma, 55\% dos adolescentes do TAI e 52,2\% do TBI completaram as 24 semanas de intervenção. Para que fosse atingido o gasto energético previsto (350kcal/sessão), as sessões de TBI foram mais prolongadas $(57 \pm 12 \mathrm{~min}$.) do que TAI ( $41 \pm 9 \mathrm{~min}$.) $(\mathrm{p}<0,001)$. As características antropométricas e da composição corporal são apresentadas na Tabela 1. Após 24 semanas de intervenção, foram verificadas reduções na massa corporal, IMC apenas para o TAI, e redução da adiposidade, bem como elevação da massa magra em ambos os grupos $(\mathrm{p}<0,001)$. Vale ressaltar que, em nenhum dos momentos analisados foram verificadas diferenças entre os grupos experimentais.

Tabela 1 - Efeitos de diferentes intensidades de treinamento aeróbio sobre variáveis antropométricas e composição corporal em adolescentes obesos submetidos à intervenção multidisciplinar.

\begin{tabular}{|c|c|c|c|c|c|}
\hline & Basal & 24 Semanas & $\begin{array}{l}\text { Efeito do } \\
\text { Grupo }\end{array}$ & $\begin{array}{c}\text { Efeito do } \\
\text { Tempo }\end{array}$ & $\begin{array}{l}\text { Efeito da } \\
\text { Interação }\end{array}$ \\
\hline \multicolumn{6}{|c|}{ Idade (anos) } \\
\hline TBI & $15,22 \pm 1,72$ & $15,89 \pm 1,72^{*}$ & \multirow[b]{2}{*}{0,92} & \multirow[b]{2}{*}{0,01} & \multirow[b]{2}{*}{0,91} \\
\hline TAI & $15,28 \pm 1,23$ & $15,95 \pm 1,23^{*}$ & & & \\
\hline \multicolumn{6}{|c|}{ Estatura $(\mathrm{cm})$} \\
\hline TBI & $163,10 \pm 8,54$ & $164,41 \pm 8,34^{*}$ & \multirow{2}{*}{0,94} & \multirow{2}{*}{0,01} & \multirow{2}{*}{0,48} \\
\hline TAI & $163,16 \pm 5,52$ & $163,90 \pm 6,25$ & & & \\
\hline \multicolumn{6}{|c|}{$M C(k g)$} \\
\hline TBI & $90,53 \pm 11,31$ & $90,34 \pm 13,76$ & \multirow{2}{*}{0,46} & \multirow{2}{*}{0,06} & \multirow{2}{*}{0,08} \\
\hline TAI & $89,56 \pm 11,70$ & $84,53 \pm 13,03 *$ & & & \\
\hline \multicolumn{6}{|c|}{ IMC (kg/m²) } \\
\hline TBI & $34,05 \pm 3,75$ & $33,40 \pm 4,40$ & \multirow{2}{*}{0,58} & \multirow{2}{*}{0,01} & \multirow{2}{*}{0,19} \\
\hline TAI & $33,60 \pm 3,84$ & $31,96 \pm 3,97^{*}$ & & & \\
\hline \multicolumn{6}{|c|}{ MG (kg) } \\
\hline TBI & $45,74 \pm 12,31$ & $32,21 \pm 10,02^{*}$ & \multirow{2}{*}{0,49} & \multirow{2}{*}{0,01} & \multirow{2}{*}{0,71} \\
\hline TAl & $43,77 \pm 7,26$ & $29,16 \pm 3,94^{*}$ & & & \\
\hline \multicolumn{6}{|c|}{ Gordura (\%) } \\
\hline TBI & $52,15 \pm 10,03$ & $38,12 \pm 6,75^{*}$ & \multirow{2}{*}{0,31} & \multirow{2}{*}{0,01} & \multirow{2}{*}{0,79} \\
\hline TAl & $49,19 \pm 7,28$ & $34,76 \pm 3,79 *$ & & & \\
\hline \multicolumn{6}{|c|}{$M M(k g)$} \\
\hline TBI & $43,77 \pm 7,38$ & $54,04 \pm 6,36^{*}$ & \multirow{2}{*}{0,59} & \multirow{2}{*}{0,01} & \multirow{2}{*}{0,59} \\
\hline TAI & $45,79 \pm 10,33$ & $55,36 \pm 10,56^{*}$ & & & \\
\hline
\end{tabular}

$\mathrm{TBI}=$ Treinamento baixa intensidade, $\mathrm{TAI}=$ Treinamento alta intensidade; $M C=$ massa corporal; $M G=$ massa gorda; $M M=$ massa magra. ${ }^{*}$ Pré $x$ Pós $(p<0,005)$ ANOVA Two Way. 
Após 24 semanas também observou-se redução nos níveis de colesterol total (9\% $\mathrm{p}=0,01)$ e $\operatorname{LDL}(17,7 \%$ - $\mathrm{p}=0,01)$ e elevação do $\operatorname{HDL}(22,8 \%$ - $\mathrm{p}=0,00)$ em resposta ao TBI. O treinamento de alta intensidade foi efetivo na redução do LDL (14,3\% - $\mathrm{p}=0,01)$ e no aumento do HDL $(28,1 \%$ - $\mathrm{p}=0,00)$ (Figura 2 ) sem alterações nas demais variáveis. Ao analisar as razões lipídicas Colesterol Total/HDL-colesterol, LDLcolesterol/HDL-colesterol, conhecidas como Índices de Castelli I e II, respectivamente, verificamos que ambos os grupos reduziram seus índices,TBI (-24,81\% Castelli I), (-33,59 \% Castelli II); TAI (- 24,45 \% Castelli I), (-31,83 \% Castelli II) (Tabela 2).

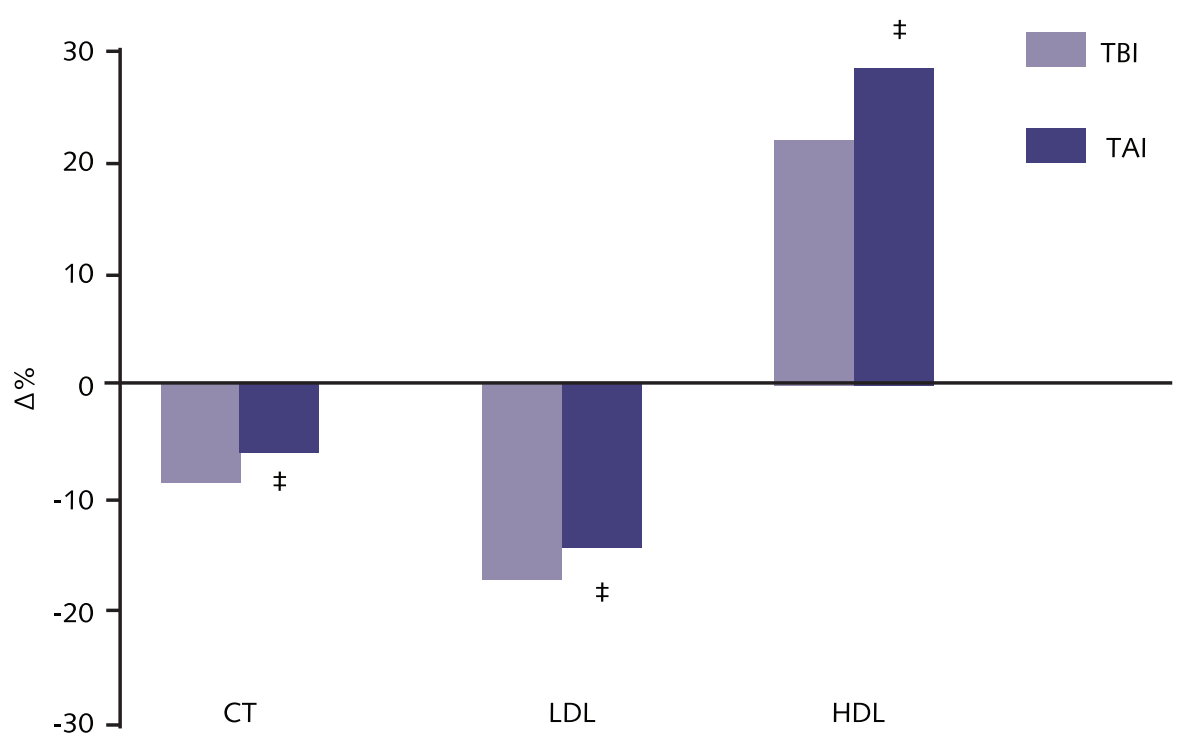

Figura 2 - Variações nas lipoproteínas de acordo com a intensidade do treinamento aeróbio. $\mathrm{CT}$ : Colesterol total, $\mathrm{LDL}=$ lipoproteína de baixa densidade, $\mathrm{HDL}=$ lipoproteína de alta densidade, $\mathrm{TBI}=$ treinamento baixa intensidade, $\mathrm{TAl}=$ treinamento alta intensidade. $\neq p<0,05$ (TBI vs. TAI).

Tabela 2 - Efeitos de diferentes intensidades de treinamento aeróbio sobre o perfil lipídico de adolescentes obesos submetidos à intervenção multidisciplinar.

\begin{tabular}{|c|c|c|c|c|c|}
\hline & Basal & 24 Semanas & $\begin{array}{c}\text { Efeito do } \\
\text { Grupo }\end{array}$ & $\begin{array}{c}\text { Efeito do } \\
\text { Tempo }\end{array}$ & $\begin{array}{l}\text { Efeito da } \\
\text { Interação }\end{array}$ \\
\hline \multicolumn{6}{|c|}{$\mathrm{CT}(\mathrm{mg} / \mathrm{dl})$} \\
\hline TBI & $161,93 \pm 35,41$ & $145,44 \pm 25,8^{*}$ & \multirow{2}{*}{0,21} & \multirow{2}{*}{0,01} & \multirow{2}{*}{0,57} \\
\hline TAI & $175,33 \pm 30,94$ & $164,60 \pm 30,20$ & & & \\
\hline \multicolumn{6}{|c|}{ LDL (mg/dl) } \\
\hline TBI & $105,15 \pm 28,43$ & $83,86 \pm 21,49 *$ & \multirow{2}{*}{0,29} & \multirow{2}{*}{0,01} & \multirow{2}{*}{0,92} \\
\hline TAI & $116,74 \pm 28,65$ & $98,53 \pm 23,23 *$ & & & \\
\hline \multicolumn{6}{|c|}{$\mathrm{HDL}(\mathrm{mg} / \mathrm{dl})$} \\
\hline TBI & $34,27 \pm 5,09$ & $41,97 \pm 4,77^{*}$ & \multirow{2}{*}{0,55} & \multirow{2}{*}{0,01} & \multirow{2}{*}{0,67} \\
\hline TAI & $32,02 \pm 8,28$ & $40,29 \pm 9,61^{*}$ & & & \\
\hline \multicolumn{6}{|c|}{ IC-1 (CT/HDL) } \\
\hline TBI & $4,81 \pm 1,44$ & $3,51 \pm 0,90^{*}$ & \multirow{2}{*}{0,20} & \multirow{2}{*}{0,01} & \multirow{2}{*}{0,92} \\
\hline TAI & $5,55 \pm 1,29$ & $4,19 \pm 0,92^{*}$ & & & \\
\hline \multicolumn{6}{|l|}{ IC-2 } \\
\hline TBI & $3,19 \pm 0,88$ & $1,88 \pm 0,49 *$ & \multirow{2}{*}{0,07} & \multirow{2}{*}{0,01} & \multirow{2}{*}{0,63} \\
\hline TAI & $3,66 \pm 0,87$ & $2,56 \pm 0,64^{*}$ & & & \\
\hline
\end{tabular}

$\mathrm{TBI}=$ Treinamento baixa intensidade, $\mathrm{TAl}=$ Treinamento alta intensidade; $\mathrm{CT}=$ colesterol total LDL=Lipoproteína de baixa densidade; $\mathrm{HDL}=$ Lipoproteína de alta densidade; $\mathrm{IC}-1=$ índice de castelli 1, IC-2 = índice de castelli 2. *Pré x Pós $(p<0,005)$ ANOVA Two Way. 


\section{DISCUSSÃO}

Os principais achados do presente estudo foram: a) redução dos níveis de LDL após 24 semanas de intervenção multidisciplinar independente da intensidade do treinamento aeróbio; b) aumento dos níveis de HDL independente da intensidade de exercício; c) redução dos níveis de colesterol total em resposta a 24 semanas de treinamento aeróbio de baixa intensidade.

Estudos abordando os efeitos do treinamento aeróbio de intensidade baixa a moderada (50\%-75\% do $\mathrm{VO}_{2 \max }$ ) têm sido bem documentados na literatura ${ }^{19,20}$, sugerindo haver intensidade e volume ideais para a realização do exercício físico, proporcionando modificações positivas no perfil lipídico.

O provável mecanismo responsável pelo aumento do HDL-c e redução do LDL-c em resposta ao exercício é o transporte reverso do colesterol. Essa via metabólica remove o excesso de colesterol da circulação e redistribui para tecidos periféricos e fígado ${ }^{21}$ Outro fator importante, é que o treinamento físico, está associado à redução da LDL oxidada e ao menor risco de aterosclerose. Mais do que isso, a prática sistematizada de exercícios físicos parece ser um importante estimulador do aumento do tamanho das moléculas de LDL-colesterol, diminuindo sua capacidade de penetrar no espaço subendotelial e ser oxidado ${ }^{22}$. Assim, mais do que um agente não farmacológico utilizado no tratamento desta doença, o exercício físico pode ser um dos pilares da prevenção primária da dislipidemia.

Neste sentido, a prática regular de exercícios físicos desde a infância e continuada na vida adulta, está associada com menor ocorrência de dislipidemia ${ }^{23}$. Além disso, a literatura aponta também que modestas reduções na massa corporal, se advindas de mudanças de estilo de vida e prática regular de exercício físico, como as verificadas no presente estudo, são efetivas na prevenção e tratamento das desordens associadas à obesidade, tanto em crianças quanto em adultos. ${ }^{24} \mathrm{De}$ acordo com as evidências, tem-se o entendimento que o treinamento físico, a princípio auxilia no controle ponderal e consequentemente na prevenção da obesidade, o que por si só, já promove melhoria do perfil lipídico ${ }^{8}$ o que indica que a relação entre exercício físico e dislipidemia reflete fundamentalmente modificações na composição corporal.

De fato, a intensidade do treinamento é um elemento chave na dosagem do exercício, e nos efeitos sobre o perfil lipídico. Neste sentido, poucos estudos ${ }^{25,26}$ relataram controle detalhado durante as sessões de modo que o efeito real da intensidade do treinamento físico, sobretudo em adolescentes, ainda não foi determinado ${ }^{12}$

Com isto, o exercício físico, independente da intensidade que é realizado é eficiente na redução da adiposidade corporal, entretanto, estudos prévios não apresentam uma padronização quanto à descrição da intensidade do esforço (percentual da frequência cardíaca, consumo máximo de oxigênio ou ainda a velocidade aeróbia máxima), o que dificulta a comparação entre os resultados ${ }^{27,28}$. Na recente revisão sistemática, Escalante et al. ${ }^{28}$ verificaram que poucos estudos usaram treinamento padronizado e controlado em esteira rolante. Adicionalmente, os programas diferiram muito em suas estruturas, durações e frequências.

Com isto, se faz importante destacar que a força do nosso estudo consiste na prescrição da intensidade do exercício, baseada no limiar anaeróbio, considerado atualmente o melhor parâmetro ventilatório para prescrição e controle da sobrecarga metabólica a qual o organismo é submetido, minimizando diferenças individuais, comumente observadas quanto a intensidade do esforço é relativa pelo $\mathrm{VO} 2 \max ^{29}$. 
O presente estudo apresenta uma limitação específica em relação à utilização de técnicas mais precisas para estimativa da composição corporal e da gordura visceral, tais como a densitometria por duplo feixe (DEXA) e ultrassonografia. Além disto, a falta de um grupo controle (sem intervenção) deve ser considerada na interpretação dos resultados aqui apresentados.

Acredita-se que o tratamento da obesidade deva ser em longo prazo, favorecendo impacto positivo sobre o controle de fatores de riscos associados à hipertensão, ao diabetes, às dislipidemias e a resistência à ação da insulina contribuindo para a melhoria na qualidade de vida de adolescentes obesos. Verifica-se, ainda, que os efeitos são dose-dependente, ou seja, podem ser ampliados à medida que aumenta o tempo de tratamento de curto para longo prazo. ${ }^{30}$

\section{CONCLUSÃO}

Os resultados do presente estudo sugerem que ambas intensidades de treinamento físico são capazes de promover alterações positivas na lipemia de adolescentes obesos submetidos a intervenção multidisciplinar, entretanto, quando comparado ao treinamento de alta intensidade, o treinamento de baixa intensidade é mais efetivo na redução do colesterol total. Vale ressaltar que mais estudos são necessários para se verificar a manutenção de tais respostas ao final da intervenção.

\section{Agradecimentos/Financiamentos}

Os autores agradecem ao CNPq e FACEPE pelo apoio financeiro para a realização deste trabalho. Agradecemos especialmente aos voluntários e seus familiares.

\section{REFERÊNCIAS}

1. Mancini MC. Obesidade e Doenças Associadas. In: Mancini MC, Geloneze B, Salles JEN, Lima JG, Carra MK. Tratado de Obesidade. Itapevi: AC Farmacêutica. 2010; 253-264.

2. Sistema de Monitoramento de Fatores de Risco e Proteção para Doenças Crônicas Não Transmissíveis por Meio de Inquérito Telefônico. VIGITEL: 2011, Brasil.http://portalsaude. saude.gov.br/portalsaude/arquivos/pdf/2012/Abr/10/vigitel_100412.pdf. [30/ jul/2011].

3. Zalesin C, Franklin BA, Miller WM, Peterson ED, Mccullough PA. Impact of obesity on cardiovascular disease. Med Clin North Am. 2011; 95(3): 919-937.

4. Al-She.hri SN, Saleh ZA, Salama MM, Hassan YM. Prevalence of hyperlipidemia among Saudi school children in Riyadh. Ann Saudi Med. 2004;24:6-8.

5. Giuliano IC, Coutinho MS, Freitas SF, Pires MM, Zunino JN, Ribeiro RQ. Lípides séricos em crianças e adolescentes da rede escolar de Florianópolis - Estudo Floripa Saudável 2040. Arq Bras Cardiol. 2005;85:85-91.

6. Kelley GA, Kelley KS, Tran ZVU. Aerobic exercise, lipids and lipoproteins in overweight and obese adults: a meta-analysis of randomized controlled trials. Int J Obes (Lond). 2005;29(8):881-93.

7. Guedes DP, Gonçalves LA. Impact of the habitual physical activity on lipid profile in adults. Arq Bras Endocrinol Metab. 2007;51(1):72-8.

8. Fernandes RA, Zanesco A. Early physical activity promotes lower prevalence of chronic diseases in adulthood. Hypertens Res. 2010; 33(9): 926-31.

9. Saakslahti A, Numminen P, Varstala V, Helenius H, Tammi A, Viikari J, et al. Physical activity as a preventive measure for coronary heart disease risk factors in early childhood. Scand J Med Sci Sports 2004;14(3):143-9.

10. Durstinni J, Crouse S, Moffat R. Lipids in exercise and sports. Energy-yelding macronutrients and energy metabolism in sport nutrition. Florida: CRC Press, 2000.

11. IV Diretriz Brasileira Sobre Dislipidemias e Prevenção da Aterosclerose Departamento de Aterosclerose da Sociedade Brasileira de Cardiologia. - Volume 88, Suplemento I, Abril 2007. 
12. Tambalis KD, Panagiotakos DB, Kavouras SA, Kallistratos AA, Moraiti IP, et al. Eleven-year prevalence trends of obesity in Greek Children: first evidence that prevalence of obesity is leveling off. Obesity. 2009; 18:161-166.

13. Tanner JM, Whithouse RH. Clinical Longitudinal standards for height, weight, weight velocity and stages of puberty. Arch Dis Child. 1976; 51:170.

14. Kuczmarski RJ, Oqden CL, Grummer-Strawn LM, Flegal KM, Guo SS, Wei R. CDC growth charts: United States. Adv Data 2000;8:1-27.

15. Slaughter MH, Lohman TG, Boileau RA, Horswill CA, Stillman, RJ, Van loan MD \& Bemben, D A. Skinfold equations for estimation of body fatness in children and youth. Hum Biol. 1988; 60:709-723.

16. Wasserman K. The anaerobic threshold measurement to evaluate exercise performance. Am Rev Respir Dis 1984;129(2):S35-40.

17. Friedewald WT, Levy RI, Fredrickson DS. Estimation of theconcentration of low-density lipoprotein cholesterol in plasma, without use of the preparative ultracentrifuge. Clin Chem. 1972;18(6): 499-502.

18. Castelli WP, Anderson K. A population at risk: prevalence of high cholesterol levels in hypertensive patients in the Framingham Study. Am J Med. 1986; 80: 23-32.

19. Johnson J, Slentz C, Houmard J, Samsa G, Duscha B, Aiken L, et al. Exercise Training amount and intensity effects on metabolic syndrome (From studies of a targeted risk reduction intercention through defined exercise). Am J Cardiol. 2007;100: 1759-1766.

20. Prado E, Dantas E. Efeitos dos exercícios aeróbio e de força nas lipoproteínas HDL,LDL e lipoproteína (a). Arq Bras Cardiol. 2002; 79(4): 429-433.

21. Leaf DA. The effect of physical exercise on reverse cholesterol transport. Metabolism. 2003; 52(8): 950-7.

22. Kraus WE, Houmard JA, Duscha BD, Knetzger KJ, Wharton MB, Mccartney JS et al. Effects of the amount and intensity of exercise on plasma lipoproteins. N Engl J Med. 2002; 347(19): 1483-92.

23. Fernandes RA, Christofaro DG, Casonatto J, Costa rosa CS, Costa FF, Freitas júnior IF, et al. Leisure time behaviors: prevalence, correlates and associations with overweight in Brazilian adults: a cross-sectional analysis. Rev Med Chile. 2010;138 (1): 29-35.

24. Garanty-Bogacka B, Syrenicz M, Goral J, Krupa B, Syrenicz J, Walczak M, et al. Changes in inflammatory biomarkers after successful lifestyle intervention in obese children. Endokrynol Pol. 2011; 62(6): 499-505.

25. Wong PC, Chia MY, Tsou IY, Wansaicheong GK, Tan B, Wang JC, et al .Effects of a 12-week exercise training programme on aerobic fitness, body composition, blood lipids and C-reactive protein in adolescents with obesity. Ann Acad Med Singapore. 2008; 37:286-293.

26. Farpour-Lambert NJ, Aggoun Y, Marchand LM, Martin XE, Herrmann FR, Beghetti M. Physical activity reduces systemic blood pressure and improves early markers of atherosclerosis in prepubertal obese children. J am Coll Cardiol. 2009;54:2396-2406.

27. Parikh T, Stratton, G. Influence of intensity of physical activity on adiposity and cardiorespiratory fitness in 5-18 year olds. Sports Med. 2011; 41(6): 477-488.

28. Escalante Y, Saavedra MJ, Dominguez MA. Improvement of the lipid profile with exercise in obese children: A systematic review. Preven Med. 2012;54:293-301.

29. Baldwin J, Snow RJ, Febbraio MA. Effect of training status and relative exercise intensity on physiological responses in men. Rev Bras Med Esporte. 2000;32(9):1648-54.

30. Tock L, Prado WL, Caranti DA, Cristofalo DM, Lederman H, Fisberg M, et al. Nonalcoholic fatty liver disease decrease in obese adolescents after multidisciplinary therapy. Eur J Forest Pathol. 2006:18(12):1241-5.

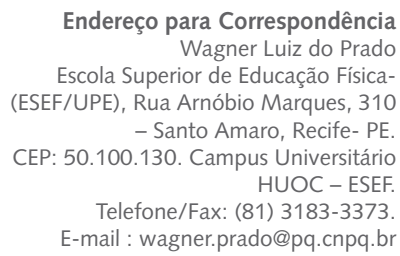

Recebido 25/11/2013

Revisado 09/01/2014

$10 / 01 / 2014$

Aprovado 11/01/2014 